\title{
Simultaneous high crystallinity and sub-bandgap optical absorptance in hyperdoped black silicon using nanosecond laser annealing
}

\section{Citation}

Franta B, Pastor D, Gandhi HH, Rekemeyer PH, Gradecak S, Aziz MJ, Mazur E. 2015.

Simultaneous high crystallinity and sub-bandgap optical absorptance in hyperdoped black silicon using nanosecond laser annealing. Journal of Applied Physics 118, no. 22.

\section{Published Version}

doi:10.1063/1.4937149

\section{Permanent link}

http://nrs.harvard.edu/urn-3:HUL.InstRepos:27416633

\section{Terms of Use}

This article was downloaded from Harvard University's DASH repository, and is made available under the terms and conditions applicable to Open Access Policy Articles, as set forth at http:// nrs.harvard.edu/urn-3:HUL.InstRepos:dash.current.terms-of-use\#OAP

\section{Share Your Story}

The Harvard community has made this article openly available.

Please share how this access benefits you. Submit a story.

\section{Accessibility}




\title{
Simultaneous high crystallinity and sub-bandgap optical absorptance in hyperdoped black silicon using nanosecond laser annealing
}

\author{
Benjamin Franta ${ }^{1, a}$, David Pastor ${ }^{1}$, Hemi H. Gandhi ${ }^{1}$, Paul H. Rekemeyer ${ }^{2}$, Silvija \\ Gradečak $^{2}$, Michael J. Aziz ${ }^{1}$, and Eric Mazur ${ }^{1}$ \\ ${ }^{1}$ School of Engineering and Applied Sciences, Harvard University, Cambridge, Massachusetts 02138, USA \\ ${ }^{2}$ Department of Materials Science and Engineering, Massachusetts Institute of Technology, Cambridge, Massachusetts \\ 02139, USA \\ a) Author to whom correspondence should be addressed. Electronic mail: bafranta@gmail.com.
}

Hyperdoped black silicon fabricated with femtosecond laser irradiation has attracted interest for applications in infrared photodetectors and intermediate band photovoltaics due to its sub-bandgap optical absorptance and light-trapping surface. However, hyperdoped black silicon typically has an amorphous and polyphasic polycrystalline surface that can interfere with carrier transport, electrical rectification, and intermediate band formation. Past studies have used thermal annealing to obtain high crystallinity in hyperdoped black silicon, but thermal annealing causes a deactivation of the sub-bandgap optical absorptance. In this study, nanosecond laser annealing is used to obtain high crystallinity and remove pressure-induced phases in hyperdoped black silicon while maintaining high sub-bandgap optical absorptance and a light-trapping surface morphology. Furthermore, it is shown that nanosecond laser annealing reactivates the sub-bandgap optical absorptance of hyperdoped black silicon after deactivation by thermal annealing. Thermal annealing and nanosecond laser annealing can be combined in sequence to fabricate hyperdoped black silicon that simultaneously shows high crystallinity, high abovebandgap and sub-bandgap absorptance, and a rectifying electrical homojunction. Such nanosecond laser annealing could potentially be applied to non-equilibrium material systems beyond hyperdoped black silicon.

\section{INTRODUCTION}

Hyperdoped black silicon is silicon that is both supersaturated with low-solubility dopants (i.e., "hyperdoped") and that possesses a light-trapping surface (the "black" in black silicon). Supersaturation with dopants increases the intrinsic optical absorptance coefficient, particularly to photon energies below the bandgap energy, $\frac{1}{}$ and a light-trapping surface increases the optical path length within the material and can lower the optical reflectance of the surface..$^{2}$ These properties in combination make hyperdoped black silicon a strong absorber of photon energies both above and below silicon's bandgap energy, including in the visible and infrared regions of the electromagnetic spectrum. The strong broadband absorptance in hyperdoped black silicon has aroused interest for its potential use in silicon-based infrared photodetectors $\underline{3}-4$ and intermediate band photovoltaics. 


\section{PREPRINT}

Various methods exist for fabricating hyperdoped black silicon, in addition to methods for fabricating silicon that is hyperdoped but optically flat and methods for fabricating black silicon that is not hyperdoped. Hyperdoped black silicon can be fabricated by irradiating silicon with femtosecond $\stackrel{6}{-}$ or nanosecond ${ }^{7}$ laser pulses in the presence of dopants, which are typically in the form of a gas $\underline{6}$ or a thin film. $\underline{8} \underline{9} \underline{10}$ Laser irradiation parameters are used that both 1) hyperdope the silicon through a process of silicon melting, dopant diffusion into the melt, and solute trapping during rapid resolidification of the silicon, and 2) produce self-organized surface structures on the nanometer and micrometer scales that have light-trapping properties. Silicon that is hyperdoped but optically flat can be fabricated by pulsed laser melting of ion implanted silicon $\underline{11} \underline{12}$ or by laser irradiation of silicon using irradiation parameters that hyperdope the silicon but avoid the formation of selforganized surface structures. $\stackrel{13}{ }$ Black silicon that is not hyperdoped can be fabricated by chemical etching of silicon, $\underline{14} \underline{15}$ by direct laser texturing, $\underline{16} \underline{17}$ or by laser irradiation of silicon using irradiation parameters that produce self-organized surface structures without the presence of dopants. $\underline{18} \underline{19}$

Some properties of flat crystalline hyperdoped silicon and hyperdoped black silicon that are important for device design have recently become better understood. First, the concentration range of chalcogen (sulfur, selenium, and tellurium) dopants that yields an intermediate band in crystalline silicon has been determined to be below 0.4 atomic $\%\left(\right.$ below $\left.2 \times 10^{20} \mathrm{~cm}^{-3}\right)$; doping above this concentration yields a metallic band structure. ${ }^{20}$ The presence of an intermediate band in hyperdoped silicon below this doping concentration is supported by density functional theory, $\frac{20}{}$ temperature-dependent conductivity, and $\mathrm{x}$-ray emission spectroscopy. $\underline{22}$ Second, carrier lifetimes, $\underline{23}$ carrier mobilities,,$\underline{24} \underline{25}$ and absorption coefficients $\underline{26} \underline{1}$ in chalcogen-hyperdoped crystalline silicon have been measured and imply that the dopant concentration that maximizes carrier extraction from a hyperdoped silicon layer of a given optical thickness is around 0.03 atomic $\%\left(1.4 \times 10^{19} \mathrm{~cm}^{-3}\right), \frac{23}{}$ which is about two orders of magnitude greater than the equilibrium solubility limit for chalcogens in silicon of 0.0001 atomic $\%$ $\left(5 \times 10^{16} \mathrm{~cm}^{-3}\right) \cdot \frac{27}{}$ Third, microstructural investigations of hyperdoped black silicon have shown that after fabrication with femtosecond laser irradiation, the light-trapping surface structures (typically cones that are 1-10 micrometers tall) are often polyphasic, containing amorphous and various pressure-induced crystalline silicon phases, and the dopants are incorporated within a thin layer on the surface which is typically $20-200 \mathrm{~nm}$ thick. $\underline{28} \underline{29} \underline{10}$ Fourth, thermal annealing increases the crystallinity of hyperdoped black silicon and can improve electrical rectification at the homojunction between the hyperdoped layer and the substrate, but thermal annealing also deactivates the sub-bandgap optical absorptance of the material in a manner consistent with the dopants diffusing to optically inactive sites in the silicon lattice (with diffusion lengths $<1$ micrometer)..$\underline{30} \underline{31}$ After deactivation, the sub-bandgap optical absorptance can be reactivated partially by heating and fast

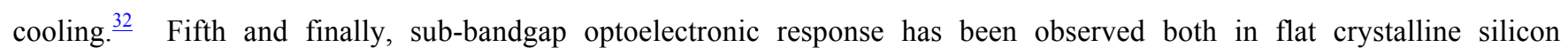




\section{PREPRINT}

hyperdoped with gold and in sulfur-hyperdoped black silicon, demonstrating that hyperdoping can indeed produce subbandgap optoelectronic response in semiconductor materials. $\underline{33} \underline{34}$

The properties of hyperdoped silicon and hyperdoped black silicon imply some design guidelines for hyperdoped optoelectronic devices. First, the dopant concentration should not exceed the upper limit of the range corresponding to intermediate band formation. Concentrations above this limit result in a metallic material with no significant optoelectronic response. Second, the thickness of the hyperdoped layer should be less than the carrier transport lengths. (The transport length for holes in sulfur-hyperdoped silicon, for example, was estimated in one study to be at least $100 \mathrm{~nm}$ under an internal field produced by the dopant concentration gradient $^{25}$ ). Because the transport length is generally less than the optical absorption depth, light trapping strategies, such as geometric light trapping from surface structures or plasmonic light trapping from metal nanoparticles, should be used to increase absorption within the hyperdoped layer. Finally, deactivation of the sub-bandgap optical absorptance from thermal annealing should be avoided in the final device material. One of the challenges in fulfilling this design guideline is to obtain high crystallinity in hyperdoped black silicon as well as high subbandgap optical absorptance, because thermal annealing (which increases the material's crystallinity) deactivates the subbandgap optical absorptance.

In this paper, we fabricate hyperdoped black silicon that is both highly crystalline and highly absorptive to sub-bandgap wavelengths by irradiating hyperdoped black silicon with nanosecond laser pulses ("ns laser annealing”). The ns laser pulses smooth and crystallize the surface, remove pressure-induced silicon phases, and maintain the material's high sub-bandgap optical absorptance and light-trapping surface morphology. Furthermore, if thermal annealing is used (e.g., to increase the electrical rectification or material crystallinity) — thereby deactivating the sub-bandgap optical absorptance — ns laser annealing can be used subsequently to reactivate the sub-bandgap optical absorptance while maintaining high crystallinity. We also find that ns laser annealing does not interfere with the rectifying homojunction in hyperdoped black silicon. Thermal annealing and ns laser annealing can be combined in series to fabricate hyperdoped black silicon that has high crystallinity, high above-bandgap and sub-bandgap optical absorptance, and a rectifying homojunction. Because nanosecond laser annealing is based on physical processes of melting and fast resolidification, it could potentially be applied to other nonequilibrium material systems beyond hyperdoped black silicon. $\underline{35} \underline{36}$

\section{EXPERIMENTAL}

We fabricated hyperdoped black silicon by irradiating a silicon wafer with fs laser pulses in the presence of sulfur hexafluoride $\left(\mathrm{SF}_{6}\right)$ gas. A monocrystalline silicon wafer (p-type, boron-doped, 7-14 ohm-cm) was placed in a vacuum 


\section{PREPRINT}

chamber which was evacuated to $<0.1$ Torr and then filled with sulfur hexafluoride gas to a pressure of 500 Torr. Femtosecond laser pulses $(800 \mathrm{~nm}, 100$-fs pulse duration, $1 \mathrm{kHz}$ repetition rate) were output by a Ti:sapphire regenerative amplifier, passed through a computer-controlled mirror galvanometer, directed into the vacuum chamber, and focused onto the silicon surface so that a pulse diameter of $0.5-1 \mathrm{~mm}$ (full width at half maximum (FWHM) of the Gaussian pulse) was obtained. Each pulse had a fluence of $8 \mathrm{~kJ} / \mathrm{m}^{2}$, and the pulses were scanned across the silicon surface (as described by Smith et al. $)^{29}$ so that each site on the surface was exposed, on average, to 200 pulses. Each time $4 \mathrm{~cm}^{2}$ of the surface was irradiated, the vacuum chamber was evacuated and refilled with new sulfur hexafluoride gas in order to prevent ablated particles from the silicon surface from accumulating in the chamber. The hyperdoped black silicon samples fabricated for this study were $1 \times 1 \mathrm{~cm}^{2}$ in area. Between batches of samples (groups of samples made on different days), we noticed some variation in the size and shape (e.g., the aspect ratio) of the resulting surface structures when using the same nominal fs laser irradiation parameters, which might result from uncertainties in estimating the fs laser pulse fluence or instabilities in our fs laser. These variations in surface structures caused variations in the optical absorptance between batches of samples. Therefore, we only directly compare samples from the same batch in optical absorptance measurements. In addition to the samples fabricated with a fs-laser pulse fluence of $8 \mathrm{~kJ} / \mathrm{m}^{2}$, we also fabricated samples with a fluence of $2.5 \mathrm{~kJ} / \mathrm{m}^{2}$, which yielded smaller conical surface structures.

We irradiated the hyperdoped black silicon with ns laser pulses. Nanosecond laser pulses were generated by ArF (193 nm) and $\mathrm{XeCl}(308 \mathrm{~nm}, 25 \mathrm{~ns}$ FWHM pulse duration) excimer lasers and a Nd:YAG laser (355 nm, 4 ns FWHM pulse duration). The laser pulse fluence was controlled by focusing the beam to increase the fluence or by reflecting a portion of the beam off of a quartz window to decrease the fluence, and the pulses were passed through a metal mask with a $2.5 \times 2.5$

$\mathrm{mm}^{2}$ square opening to improve the uniformity of the pulse intensity profile, resulting in uniform pulses with fluences of $0.6-$ $2.2 \mathrm{~J} / \mathrm{cm}^{2}$. The laser pulses were scanned across the hyperdoped black silicon surface in ambient air. Typically, each site on the hyperdoped black silicon surface was exposed to a single nanosecond laser pulse, though exposures of up to 40 pulses were carried out to determine the effects of multiple exposures.

We measured the crystallinity of the hyperdoped black silicon surface using Raman spectroscopy. For Raman spectrum measurements, a $10 \mathrm{~mW}$ HeNe laser beam $(632.8 \mathrm{~nm})$ was passed through a $50 \times$ objective lens (resulting in a spot diameter of 5 micrometers on the hyperdoped black silicon surface) and projected onto a CCD using a diffraction grating with 1200 grooves/mm, producing Raman spectra with a spectral wavenumber resolution of $2.0 \mathrm{~cm}^{-1}$. After subtracting a baseline from the measured spectrum, the relative crystallinity of the hyperdoped black silicon surface was determined by dividing the area under the spectral curve from $470-490 \mathrm{~cm}^{-1}$ (containing the broad transverse-optical vibrational mode of amorphous silicon 


\section{PREPRINT}

centered at $480 \mathrm{~cm}^{-1} \frac{37}{\text { }}$ ) by the area under the spectral curve from $515-525 \mathrm{~cm}^{-1}$ (containing the sharp optical vibrational mode of crystalline silicon at $520 \mathrm{~cm}^{-1} \stackrel{38}{)}$. We also measured the width (FWHM) of the crystalline silicon peak at $520 \mathrm{~cm}^{-1}$. Results from pristine monocrystalline silicon wafers were used as references. Measurements from each sample were averaged between 100 spectra collected within a $15 \times 15 \mu \mathrm{m}^{2}$ grid in order to account for possible variations over the surface.

We investigated the surface morphology of the hyperdoped black silicon using a field emission scanning electron microscope (FESEM). Samples were imaged at an angle of 45 degrees without foreshortening correction, using an accelerating voltage of $3 \mathrm{kV}$ and a working distance of $16 \mathrm{~mm}$.

We investigated the microstructure of the hyperdoped black silicon using cross-sectional bright field transmission electron microscopy (BF-TEM) and selected area diffraction (SAD). TEM samples were prepared by lift-out in a dual-beam FIB/SEM. Protection layers of carbon and platinum were deposited in situ prior to lift-out. Thinning steps were performed at $30 \mathrm{kV}$ with the final thinning at $5 \mathrm{kV}$, which has been shown to leave $2.5 \mathrm{~nm}$ of amorphous surface damage in silicon. $\frac{39}{}$ TEM and SAD were performed with a field emission TEM operated at $200 \mathrm{kV}$. For imaging of bend contours, BF-TEM images were obtained several degrees off the [110] zone axis with an objective aperture to enhance diffraction contrast.

We measured the optical absorptance of the hyperdoped black silicon using an ultraviolet-visible-near infrared (UV-VISNIR) spectrophotometer (for wavelengths of 0.4-2.5 $\mu \mathrm{m}$ ) and a Fourier transformed infrared (FTIR) spectrometer (for wavelengths of 2.4-10.0 $\mu \mathrm{m})$. Reflectance $(R)$ and transmittance $(T)$ spectra were measured with integrating spheres and were used to calculate the optical absorptance $(A=1-R-T)$. The FTIR spectra were matched to the spectrophotometer spectra in the overlapping wavelength range $(2.4-2.5 \mu \mathrm{m})$.

We measured the current-bias behavior of the hyperdoped black silicon with a probe station and parametric analyzer. To apply metal electrodes to the samples, we cleaned the samples in solvents (acetone, isopropanol, and water), defined four $2 \times 2$ $\mathrm{mm}^{2}$ square regions with photoresist on the hyperdoped black silicon surface, carried out a plasma clean to remove organic debris $\left(75 \mathrm{~W}, 40 \mathrm{sec}\right.$, $\left.40 \mathrm{sccm} \mathrm{O}_{2}\right)$, dipped the sample in hydrofluoric acid $(\mathrm{HF})$ to remove the native surface oxide $(5 \% \mathrm{HF}$ solution, $1 \mathrm{~min}$.), used electron beam evaporation to deposit $\mathrm{Ti}$, Ni, and $\mathrm{Ag}(20 \mathrm{~nm}, 20 \mathrm{~nm}$, and $200 \mathrm{~nm}$ thicknesses, respectively) on the hyperdoped black silicon surface in sequence, used thermal evaporation to deposit Al (200 nm thickness) on the silicon wafer surface reverse of the hyperdoped black silicon surface, lifted off the remaining photoresist in heated acetone (80 deg. C), and cleaned the samples in solvents (acetone, isopropanol, and water). After electrode deposition, rapid thermal annealing (200-500 deg. C., 30 sec., $\mathrm{N}_{2}$ atmosphere) was used to obtain ohmic contact between the electrodes and the silicon surface. To measure the current-voltage behavior of the hyperdoped black silicon, samples were placed on a metal chuck (with the Al electrode in contact with the chuck), and a tungsten probe was placed in contact with one of the Ti-Ni-Ag 


\section{PREPRINT}

electrodes on the hyperdoped black silicon surface. The bias between the chuck and the probe was swept from $-20 \mathrm{~V}$ to +20 $\mathrm{V}$ and the resulting current was measured. The four electrodes on each hyperdoped black silicon sample were used to check the consistency of the measurements. A pristine silicon wafer with Al electrodes on both sides was used as a reference.

We used thermal annealing to crystallize the hyperdoped black silicon and deactivate its sub-bandgap optical absorptance. Samples were thermally annealed in a tube furnace at $700 \mathrm{deg}$. $\mathrm{C}$ for $30 \mathrm{~min}$. in forming gas $\left(95 \% \mathrm{~N}_{2}, 5 \% \mathrm{H}\right.$, $300 \mathrm{sccm})$.

To test the effects of combining thermal annealing and ns laser annealing in sequence, we fabricated hyperdoped black silicon with fs laser irradiation, thermally annealed the sample (700 deg. C., 30 min., forming gas atmosphere), performed ns laser annealing (355 nm Nd:YAG pulse, $1.1 \mathrm{~J} / \mathrm{cm}^{2}$ fluence), deposited metal electrodes onto the sample, rapidly thermally annealed the sample (500 deg. C., 30 sec., $\mathrm{N}_{2}$ atmosphere), and performed ns laser annealing again. Between each step, we measured the sample's crystallinity and current-bias behavior. We also measured the optical absorptance between each step on an identical sample that underwent the same process without deposition of metal electrodes, which would have interfered with the optical absorptance measurements.

\section{RESULTS}

Fabrication with a fs-laser pulse fluence of $8 \mathrm{~kJ} / \mathrm{m}^{2}$ yielded hyperdoped black silicon with a rough surface morphology consisting of nm-scale granular structures overlying $\mu \mathrm{m}$-scale conical structures, as shown in Figure 1. The nm-scale granular structures might have originated as particulate debris generated by laser ablation and deposited onto the silicon surface during the fs laser irradiation process. $\underline{40} \underline{41}$ The $\mu \mathrm{m}$-scale conical structures in our hyperdoped black silicon were about $10 \mu \mathrm{m}$ tall and $5 \pm 2 \mu \mathrm{m}$ wide at the base, with variation between individual cones. The cones had an elliptical base, perhaps due to the linear polarization of the laser beam, with the base width about twice as great in one dimension as in the orthogonal dimension. BF-TEM images showed a thin, 50-100 nm skin layer of amorphous material on the surface of the conical structures as well as contrast-producing features within the conical structures, which may be small pockets of amorphous silicon. ${ }^{28}$ Consistent with previous studies, the hyperdoped black silicon showed amorphous silicon and pressureinduced crystalline silicon phases in Raman spectra, $\underline{28} \underline{29}$ near-unity optical absorptance of above- and below-bandgap photon energies, $\frac{30}{-}$ and deactivation of sub-bandgap absorptance after thermal annealing. $\underline{30}$

Nanosecond laser annealing of hyperdoped black silicon resulted in nm-scale smoothing of the surface, leaving the 10$\mu \mathrm{m}$-scale conical structures intact, as shown in Figure 1. After ns laser annealing, diffraction contrast imaging and SAD 


\section{PREPRINT}

indicated that the surface was single-crystalline. Nanosecond laser annealing also removed most of the contrast-producing features within the conical structures.
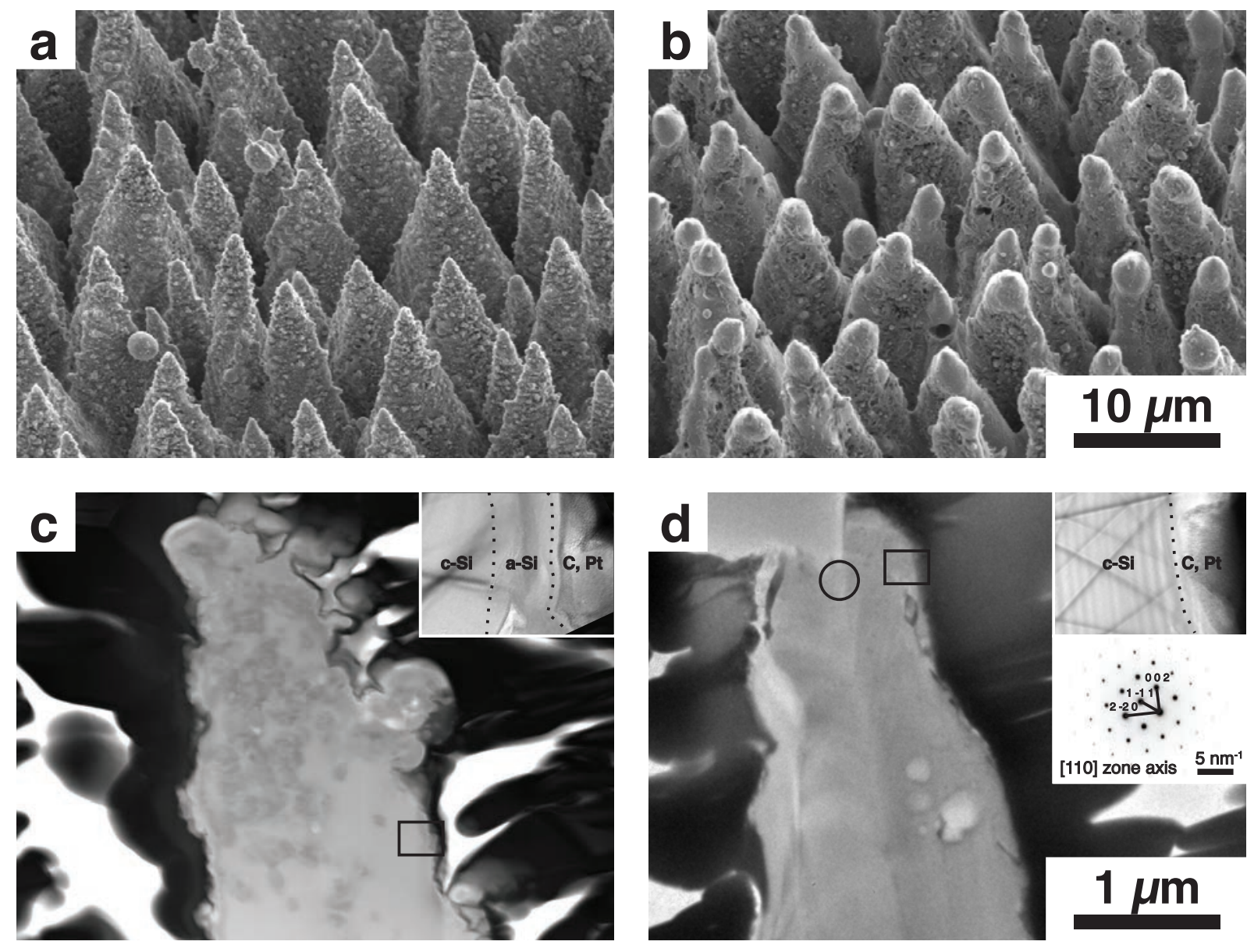

FIG. 1. (A) Hyperdoped black silicon (fabricated with a fs-laser pulse fluence of $8 \mathrm{~kJ} / \mathrm{m}^{2}$ ) with nm-scale granular structures overlying conical structures about $10 \mu \mathrm{m}$ in height and $5 \pm 2 \mu \mathrm{m}$ in width. (Image obtained at 45 -degree angle without foreshortening correction.) (B) Nanosecond laser annealing (here, with a fluence of $2.2 \mathrm{~J} / \mathrm{cm}^{2}$ ) causes smoothing of the $\mathrm{nm}$-scale granular structures while keeping the 10$\mu \mathrm{m}$-scale conical structures intact. (C) Cross-section of a conical surface structure before ns laser annealing shows nm-scale surface roughness and contrast-producing pockets within the conical surface structure. BF-TEM imaging of the surface with enhanced diffraction contrast (inset indicated by box $300 \mathrm{~nm}$ wide) shows an amorphous skin (a-Si) 50-100 nm thick between the crystalline core (c-Si) and the protective carbon and platinum layers $(\mathrm{C}, \mathrm{Pt})$. In this imaging mode, bend contours are observed in the crystalline core but do not appear in the amorphous skin. (D) Cross-section of a conical surface structure after ns laser annealing (again, with a fluence of $2.2 \mathrm{~J} / \mathrm{cm}^{2}$ ) shows smoothing of nm-scale surface roughness and removal of contrast-producing pockets within the conical surface structure. BF-TEM imaging of the surface (upper inset indicated by box $300 \mathrm{~nm}$ wide) shows crystalline material throughout. Selected area electron diffraction (lower inset indicated by circled area) indicates that the core is single crystalline ([110] zone axis).

Nanosecond laser annealing removed the amorphous and pressure-induced crystalline silicon phases shown in Raman spectra, as shown in Figure 2. $\frac{28}{2}$ The lowest ns pulse fluence we investigated $\left(0.6 \mathrm{~J} / \mathrm{cm}^{2}\right)$ was adequate to remove the pressure-induced crystalline silicon phases completely. The amount of amorphous silicon shown in Raman spectra decreased as the ns pulse fluence was increased, with pulse fluences greater than about $1.0 \mathrm{~J} / \mathrm{cm}^{2}$ yielding nearly undetectable amounts of amorphous silicon, shown in Figure 3. The decrease in amorphous silicon did not depend on the number of pulses (1-40 


\section{PREPRINT}

pulses), the pulse wavelength (193, 308, or $355 \mathrm{~nm}$ ), or the pulse duration (4 or $25 \mathrm{~ns})$. Nanosecond laser annealing also reduced the width (FWHM) of the crystalline silicon peak at $520 \mathrm{~cm}^{-1}$ by about $1 \mathrm{~cm}^{-1}$, as shown in Figure 3 inset. For example, the FWHM of the crystalline silicon peak was reduced from $8.4 \pm 0.3 \mathrm{~cm}^{-1}$ (before ns laser annealing) to $7.3 \pm 0.3 \mathrm{~cm}^{-}$

${ }^{1}$ (after ns laser annealing at fluences above $1.5 \mathrm{~J} / \mathrm{cm}^{2}$ ). Even after ns laser annealing, the peak width remained greater than that measured from pristine monocrystalline silicon $\left(6.8 \pm 0.1 \mathrm{~cm}^{-1}\right)$. The peak width we measured in monocrystalline silicon was greater than values reported in the literature, $\frac{38}{}$ which could be due to the low spectral resolution of our Raman measurements. Nonetheless, we consistently observed a reduction in peak width after ns laser annealing of about $1 \mathrm{~cm}^{-1}$.

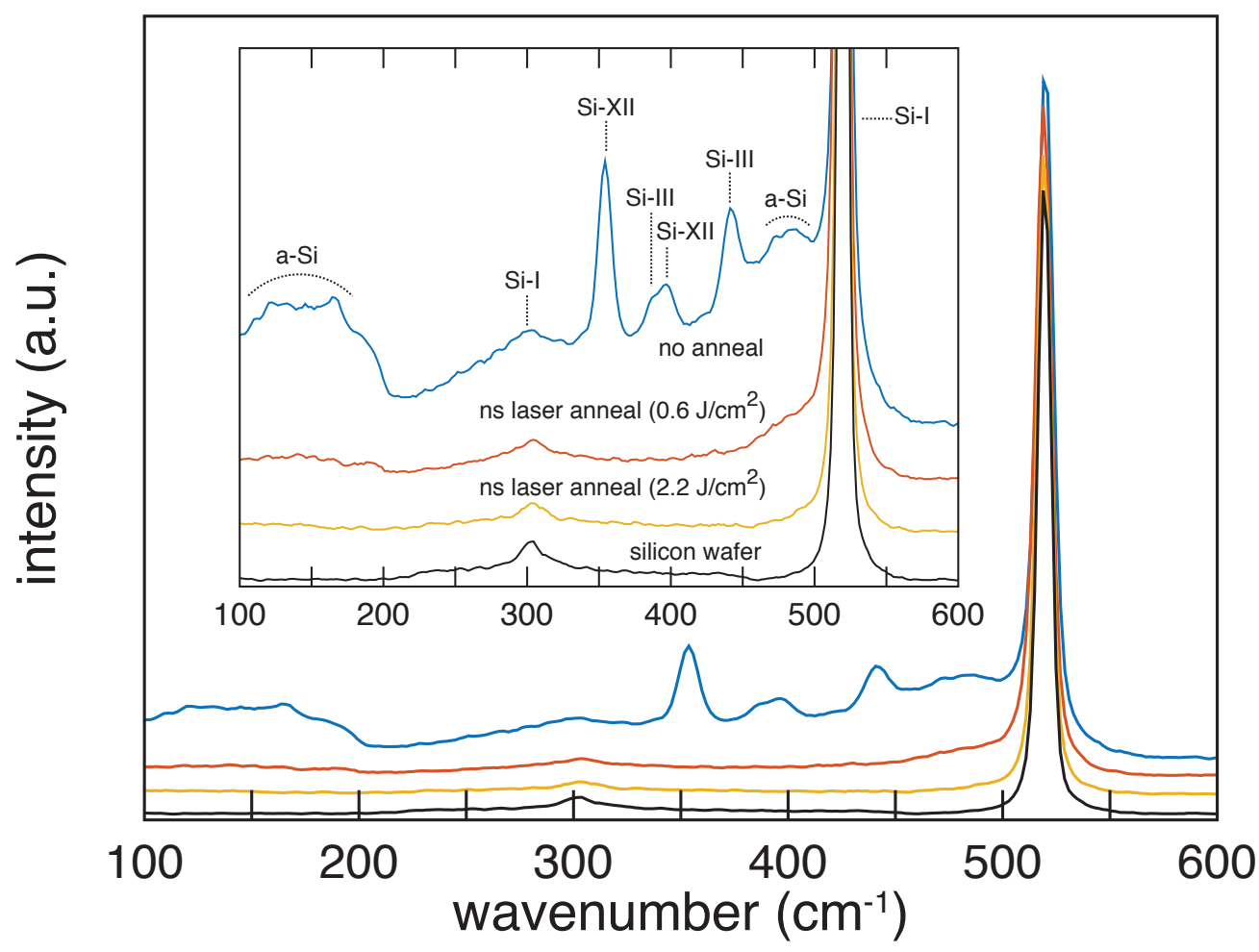

FIG. 2. (Color online) Stokes Raman spectra of hyperdoped black silicon (fabricated with a fs-laser pulse fluence of $8 \mathrm{~kJ} / \mathrm{m}^{2}$ ) before and after ns laser annealing (normalized and offset to show individual spectra). The rescaled inset highlights Raman modes corresponding to amorphous silicon (a-Si), pressure-induced crystalline phases ( $\mathrm{Si}-\mathrm{III}$ and $\mathrm{Si}-\mathrm{XII})$, and conventional crystalline silicon (Si-I). ${ }^{-2}$ Nanosecond laser annealing at low fluence $\left(0.6 \mathrm{~J} / \mathrm{cm}^{2}\right)$ removed the pressure-induced crystalline phases and much of the amorphous silicon, while ns laser annealing at high fluence $\left(2.2 \mathrm{~J} / \mathrm{cm}^{2}\right)$ removed the pressure-induced crystalline phases and nearly all of the amorphous silicon. 
PREPRINT

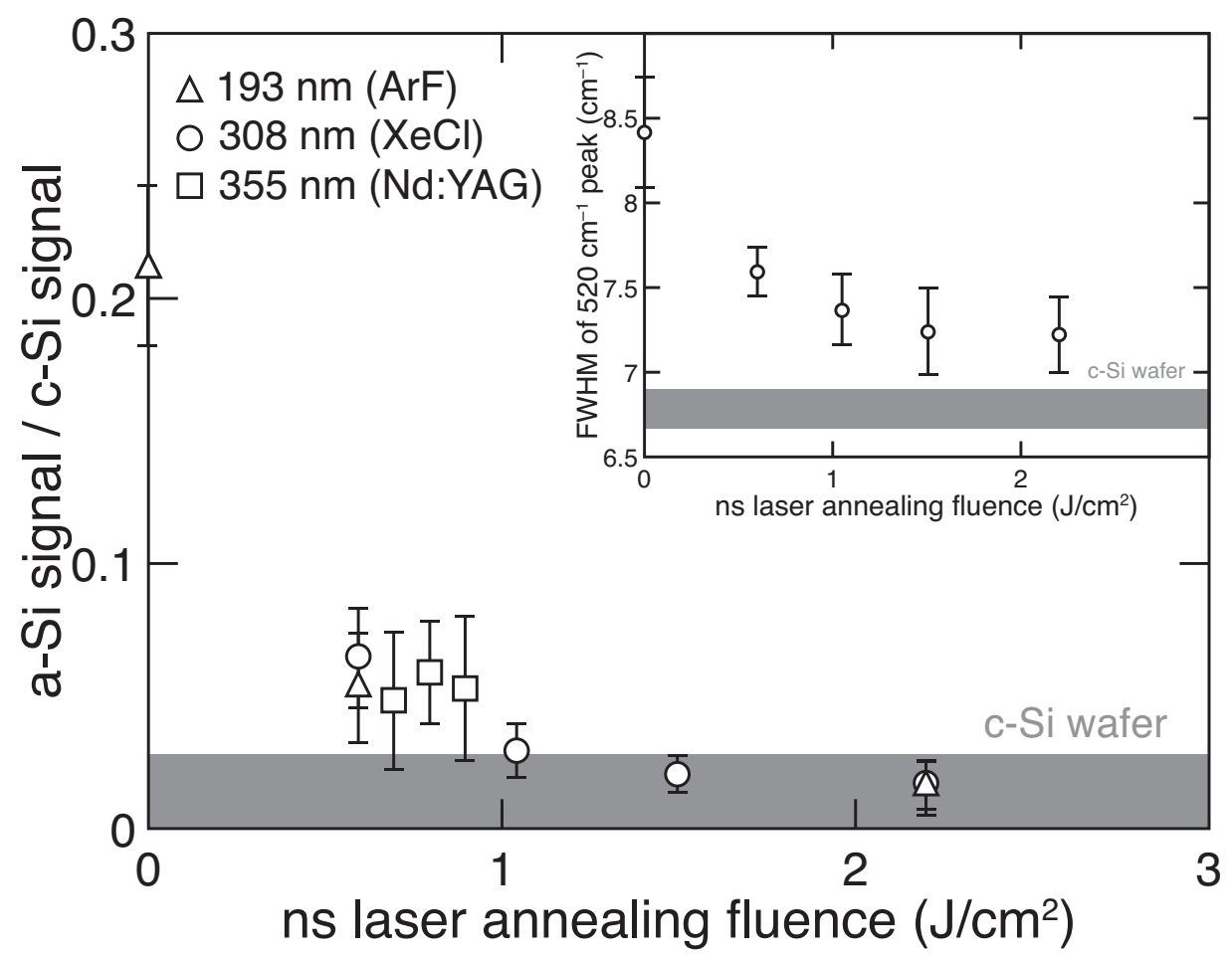

FIG. 3. Raman spectra indicate that ns laser annealing can increase the crystallinity of hyperdoped black silicon (fabricated with a fs-laser pulse fluence of $8 \mathrm{~kJ} / \mathrm{m}^{2}$ ) near to that of monocrystalline silicon (gray box). The amount of crystallization increases with the ns laser pulse fluence. Additionally, the width (FWHM) of the crystalline silicon Raman peak at $520 \mathrm{~cm}^{-1}$ decreases with increasing ns laser pulse fluence (inset).

The optical absorptance of hyperdoped black silicon, including its sub-bandgap optical absorptance, remained high after ns laser annealing, as shown in Figure 4. For example, after ns laser annealing with a pulse fluence of $2.2 \mathrm{~J} / \mathrm{cm}^{2}$ (the highest fluence we investigated), the above-bandgap absorptance (which was about 95\%) remained unchanged, and the sub-bandgap absorptance (which was greater than 90\%) increased slightly. 
PREPRINT

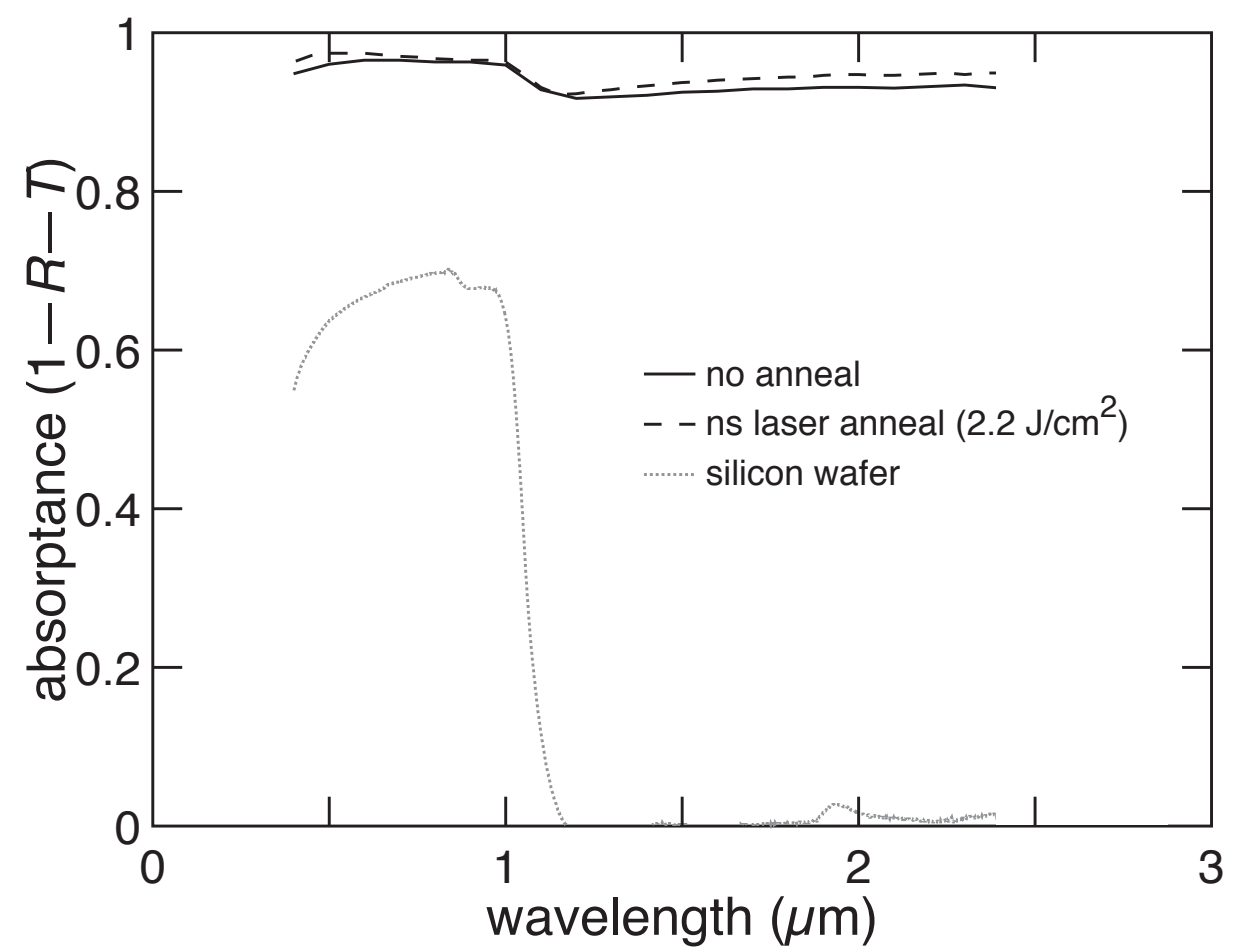

FIG. 4. Nanosecond laser annealing (here, with a fluence of $2.2 \mathrm{~J} / \mathrm{cm}^{2}$ ) preserves the high above-bandgap and sub-bandgap optical absorptance produced by the light-trapping surface as long as the surface structures are sufficiently large (here, around $10 \mu \mathrm{m}$ tall and $5 \pm 2$ $\mu \mathrm{m}$ wide at the base).

Obtaining consistent current-bias measurements required rapid thermal annealing at $500{ }^{\circ} \mathrm{C}$ for $30 \mathrm{sec}$. after electrode deposition. This rapid thermal annealing shifted the diode turn-on voltage (defined as the point of greatest curvature in the current-bias curve) from $0.2 \pm 0.1 \mathrm{~V}$ to $0.5-1.5 \mathrm{~V}$ (with some variation between samples), shown in Figure 5. Rapid thermal annealing before electrode deposition had no effect on the current-bias measurements. Rapid thermal annealing did not decrease the presence of amorphous silicon, but it did decrease the sub-bandgap optical absorptance, with higher annealing temperatures decreasing the absorptance further. Nanosecond laser annealing did not affect electrical current-bias behavior, as shown in Figure 5. Rectification ratios of $50 \pm 20$ at $\pm 10 \mathrm{~V}$ were obtained. 


\section{PREPRINT}

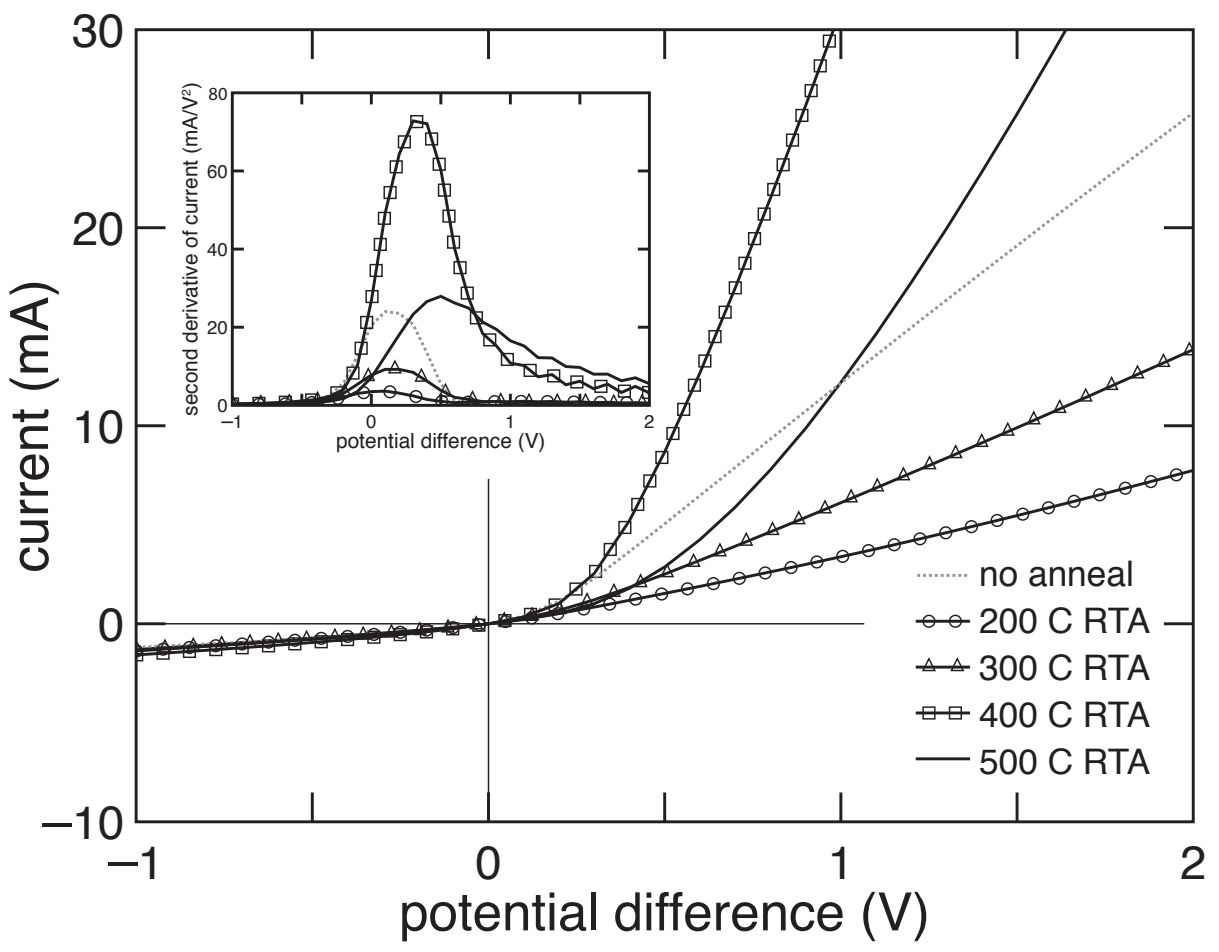

FIG. 5. The turn-on voltage of hyperdoped black silicon diodes (fabricated with a fs-laser pulse fluence of $8 \mathrm{~kJ} / \mathrm{m}^{2}$ ) is increased by rapid thermal annealing (RTA) after electrode deposition. Higher rapid thermal annealing temperatures up to $500 \mathrm{deg}$. C (for $30 \mathrm{sec}$.) produce higher turn-on voltages (inset). Turn-on voltages of around $0.2 \mathrm{~V}$ are typical of Schottky diodes, $\stackrel{42}{-}$ while turn-on voltages of around $0.7 \mathrm{~V}$ are typical of silicon homojunction diodes. $\underline{43}$

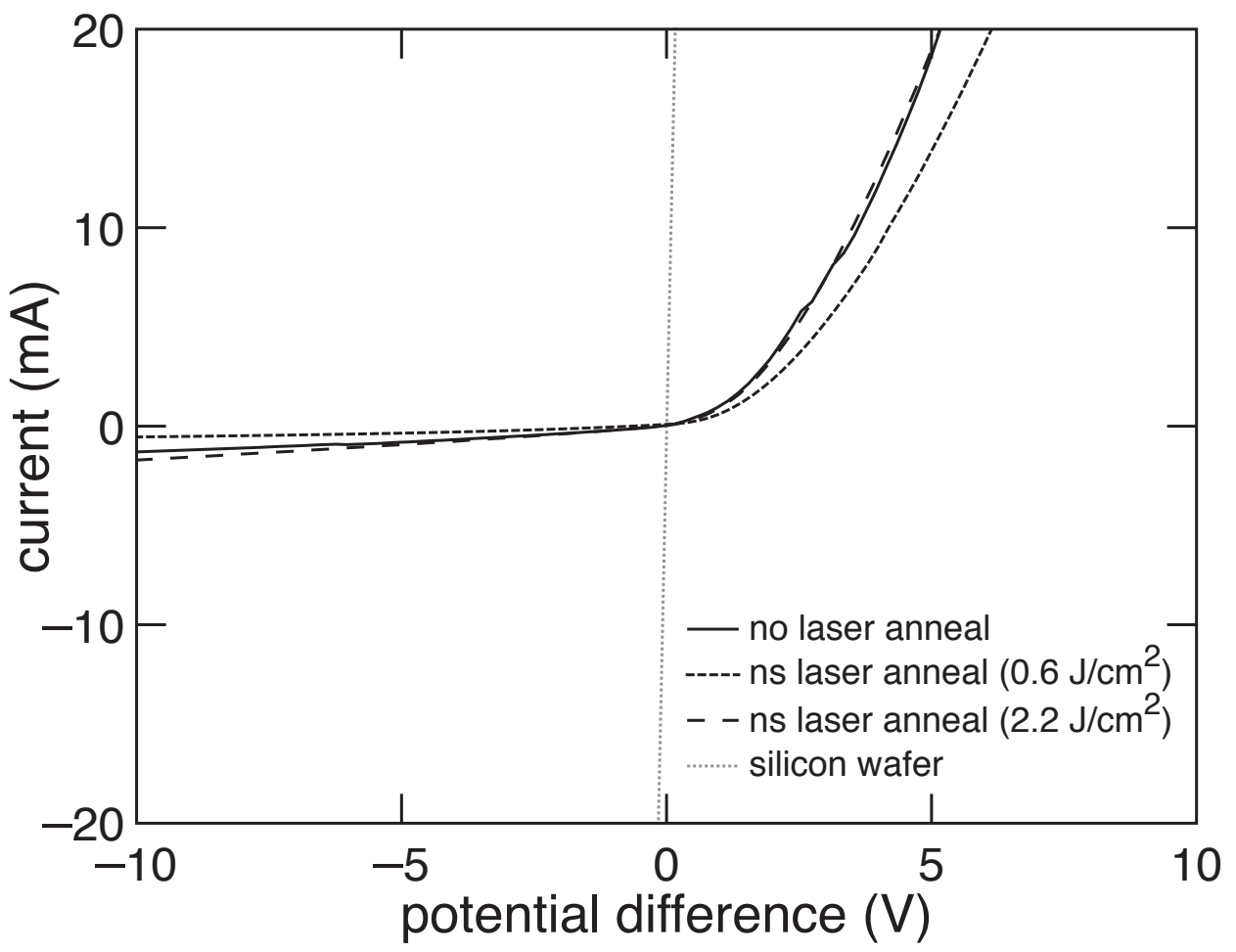

FIG. 6. Electrical rectification in hyperdoped black silicon diodes (fabricated with a fs-laser pulse fluence of $8 \mathrm{~kJ} / \mathrm{m}^{2}$ ) is maintained after ns laser annealing. All diodes underwent rapid thermal annealing (RTA) at $500 \mathrm{deg}$. C (for $30 \mathrm{sec}$.) after electrodes were deposited. 


\section{PREPRINT}

After thermal annealing deactivated the sub-bandgap optical absorptance, ns laser annealing reactivated the sub-bandgap optical absorptance near to its original level. For example, after thermal annealing at 700 deg. C for 30 min., the subbandgap optical absorptance (1.2-4.0 $\mu \mathrm{m}$ wavelengths) was reduced from about $80 \%$ to about $20 \%$, as shown in Figure 7 , and the amount of amorphous silicon was reduced to an undetectable level. Subsequent ns laser annealing with a pulse fluence of $1.1 \mathrm{~J} / \mathrm{cm}^{2}$ restored the sub-bandgap optical absorptance to $70-80 \%$ (with a broad sub-bandgap absorption peak at a wavelength of $2.0 \pm 0.8$ micrometers) while maintaining high crystallinity. Subsequently, rapid thermal annealing reduced the sub-bandgap absorptance to about 60\%. Finally, ns laser annealing again restored the sub-bandgap absorptance to $70-80 \%$. The samples in Figure 7 exhibit lower optical absorptance than the samples in Figure 4 due to small variations in surface structure size between batches of samples (see Experimental Section). As a result, we only directly compare samples from the same batch.

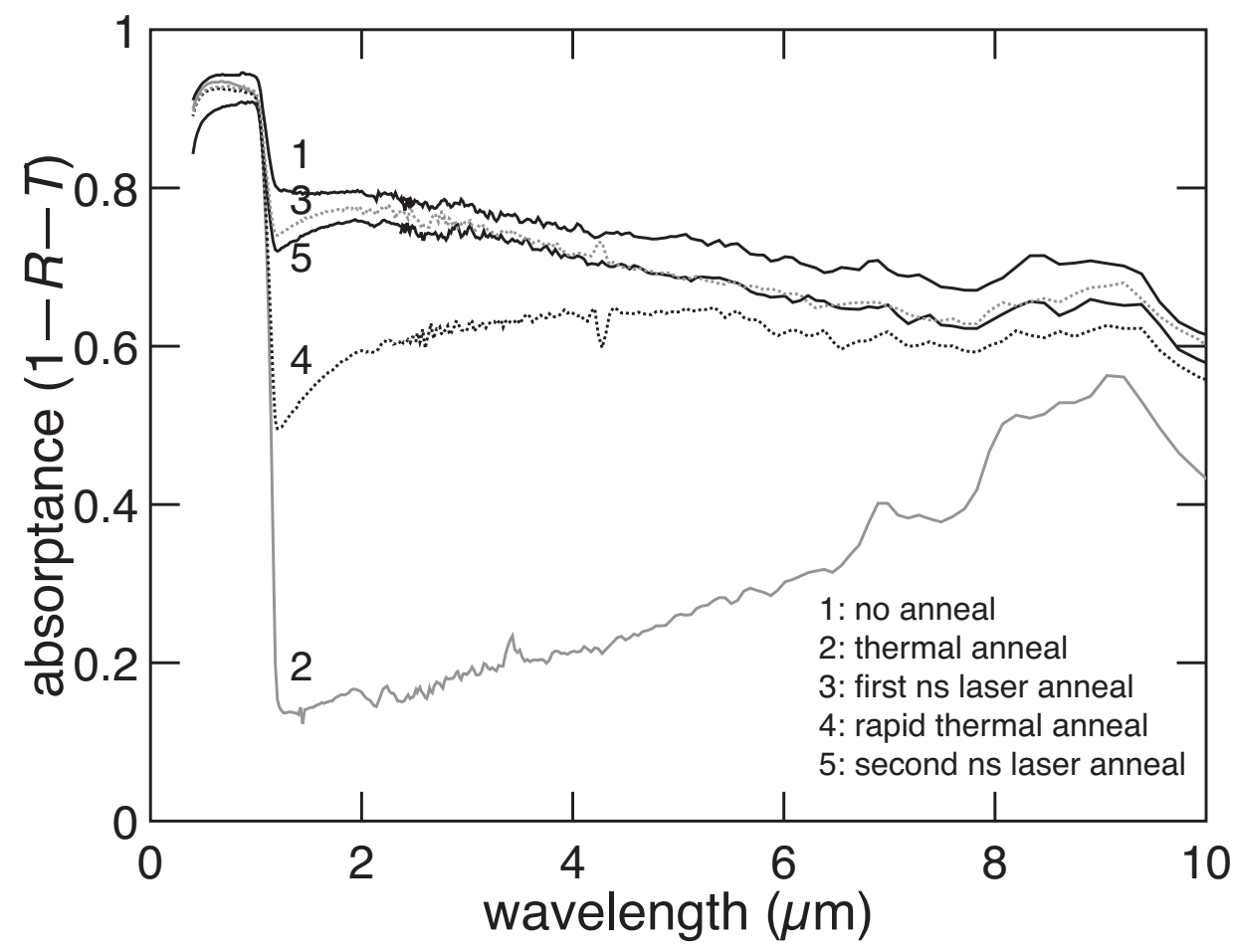

FIG. 7. The effects of thermal and ns laser annealing in sequence on the optical absorptance. 1) Femtosecond-laser-fabricated hyperdoped black silicon exhibits high above-bandgap and sub-bandgap optical absorptance. 2) Thermal annealing (700 deg. C, 30 min.) crystallizes the hyperdoped black silicon but deactivates its sub-bandgap optical absorptance. 3) Nanosecond laser annealing (here, with a fluence of $1.1 \mathrm{~J} / \mathrm{cm}^{2}$ ) maintains the high crystallinity while reactivating the sub-bandgap optical absorptance near to its original level. 4) Rapid thermal annealing (500 deg. C, $30 \mathrm{sec}$.) after depositing electrodes again deactivates (partially) the sub-bandgap optical absorptance. 5) Nanosecond laser annealing again reactivates the sub-bandgap optical absorptance near to its original level. The final hyperdoped black silicon diodes are highly crystalline, highly optically absorbing, and electrically rectifying. 


\section{PREPRINT}

Fabrication with a fs-laser pulse fluence of $2.5 \mathrm{~kJ} / \mathrm{m}^{2}$ yielded hyperdoped black silicon with smaller conical surface structures, which were about $1 \mu \mathrm{m}$ in size (see supplementary figure $\mathrm{S} 1$ ). $\stackrel{44}{ }$ Before ns laser annealing, these samples exhibited amorphous and pressure-induced crystalline phases similar to unannealed hyperdoped black silicon with $10-\mu \mathrm{m}$ surface structures (as shown in Figure 1 and Figure 3), above-bandgap optical absorptance of 90\%, and sub-bandgap optical absorptance of $80 \%$ (see supplementary figure S2). $\stackrel{44}{=}$ After ns laser annealing with a fluence of $0.6 \mathrm{~J} / \mathrm{cm}^{2}$, the $1-\mu \mathrm{m}$ conical surface structures remained intact, the amorphous and pressure-induced crystal phases were reduced to an undetectable level, and the sub-bandgap optical absorptance increased to $80-90 \%$. After ns laser annealing with a higher fluence of $2.2 \mathrm{~J} / \mathrm{cm}^{2}$, the conical surface structures were largely destroyed, the above-bandgap optical absorptance dropped to 80-90\%, and the sub-bandgap optical absorptance dropped to $60 \%$ (the amorphous and pressure-induced crystal phases were again reduced to an undetectable level).

\section{DISCUSSION}

Nanosecond laser annealing produces three notable effects: smoothing the surface on the nm scale, converting amorphous and pressure-induced silicon phases to conventional crystalline silicon (Si-I), and placing or maintaining the dopant atoms in optically active, non-equilibrium positions. These effects are analogous to the effects of ns pulsed laser melting on flat silicon hyperdoped by ion implantation, which also include crystallizing the surface and placing or maintaining the dopant atoms in optically active, non-equilibrium positions. In flat silicon hyperdoped by ion implantation, a series of ns laser pulses can be used to melt the surface (which is amorphous after ion implantation) to a depth reaching the non-hyperdoped, crystalline silicon substrate. Resolidification of the melt initiates from the substrate-liquid interface, moves toward the surface (under some conditions epitaxially), and traps the dopant atoms in optically active, non-equilibrium positions within the crystal, producing crystalline hyperdoped silicon. The key to obtaining crystalline hyperdoped silicon that exhibits sub-bandgap optical absorptance is to produce a resolidification velocity that is less than the amorphization velocity of silicon $(15 \mathrm{~m} / \mathrm{s} \stackrel{45}{)})$ but still sufficiently high to produce solute trapping. $\frac{12}{}$ With this method, a thin layer $(e . g ., 200$ $\mathrm{nm}$ ) of single-crystal hyperdoped silicon can be obtained. Processes that can interfere with the formation of single-crystal hyperdoped silicon include cellular breakdown (which can result in columnar crystals separated by dopant-rich boundaries) $\underline{46}$

$\underline{47}$ and explosive crystallization of amorphous silicon (which can result in polycrystalline silicon containing nanometer-scale grains). $\stackrel{48}{-}$ The physical processes that occur during ns laser annealing of hyperdoped black silicon appear similar to but more complex than these processes that occur during ns pulsed laser melting of flat hyperdoped silicon. 


\section{PREPRINT}

From cross-sectional TEM images of the conical surface structures (Figure 1), we estimate that after fabrication with fs laser irradiation, the surface is covered with a 50-100 nm skin of amorphous, sulfur-bearing silicon. Below this skin layer and within the conical surface structures, contrast-producing features are visible, which could be pockets of amorphous silicon within the crystalline silicon matrix, as indicated by previous studies. $\stackrel{28}{ }$ The removal of these contrast-producing features could be the result of heat diffusion into the conical surface structures during ns laser annealing. We infer that after ns laser annealing, the black silicon surface contains a skin of crystalline hyperdoped silicon, because BF-TEM imaging, SAD, and Raman spectra all indicate that the surface is crystalline, and the strong sub-bandgap optical absorptance indicates that the surface is hyperdoped. In future work, it would be interesting to investigate the effect of ns laser annealing on the distribution of dopant atoms by using, for example, three-dimensional atom probe or other tomographic methods. ${ }^{49}$

We estimate the melting depth from ns laser annealing with a fluence of $1.0 \mathrm{~J} / \mathrm{cm}^{2}$ to be about $100 \mathrm{~nm}$ on hyperdoped black silicon with $10-\mu \mathrm{m}$-scale structures, because the amorphous skin on the surface after fabrication with fs laser irradiation appears to be $50-100 \mathrm{~nm}$ thick, and after ns laser annealing at fluences above $1.0 \mathrm{~J} / \mathrm{cm}^{2}$, little-to-no amorphous silicon is detected (Figure 3). To verify this estimate, we compare it to the average melting depth expected from geometric optics. We stress that the laser intensity at the surface might be very inhomogeneous due to surface reflections and other effects. We do not discuss these effects in detail here; our aim, rather, is to determine whether $100 \mathrm{~nm}$ is a reasonable estimate for the average melting depth. The melting threshold fluence for ns laser irradiation of flat amorphous silicon (248 nm wavelength) has been reported as $0.1-0.2 \mathrm{~J} / \mathrm{cm}^{2}, \underline{50} \underline{51} \underline{52}$ which we have verified in laser-induced melting simulations. However, the conical surface structures increase the surface area of black silicon relative to flat silicon and thus reduce the average effective fluence of the laser pulse, in this case by a factor of about 4 (because surface cones with a height of $10 \mu \mathrm{m}$ and a diameter of $5 \mu \mathrm{m}$ increase the surface area by a factor of 4). At the same time, the surface reflectance of the amorphous silicon is decreased from $0.6 \frac{52}{5}$ to 0.1 due to light-trapping, indicating that about twice as much energy is absorbed by a hyperdoped black silicon surface than by a flat amorphous silicon surface for a given laser pulse fluence. Therefore, we estimate an average melting threshold fluence for our hyperdoped black silicon of $0.2-0.4 \mathrm{~J} / \mathrm{cm}^{2}$, and that a ns laser pulse fluence of 1.0 $\mathrm{J} / \mathrm{cm}^{2}$ on the hyperdoped black silicon we examined produces an average melting depth similar to that produced by a ns laser pulse with a fluence of $0.25-0.5 \mathrm{~J} / \mathrm{cm}^{2}$ on flat amorphous silicon. Indeed, reported simulations and experiments indicate that

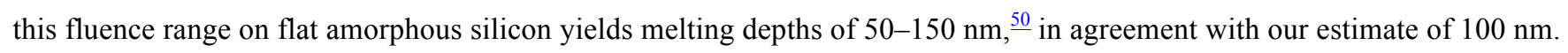
The melting depths at the peaks of the conical surface structures and in the valleys between the structures could be expected to be greater than our estimate, as these surfaces are closer to normal incidence to the ns laser pulses than are the side walls of the conical surface structures. Indeed, the most prominent melting visible in SEM images of the surface after ns laser 


\section{PREPRINT}

annealing appears at the peaks of the surface structures. Because crystalline silicon has a higher melting threshold than amorphous silicon $\left(0.7-0.8 \mathrm{~J} / \mathrm{cm}^{2}\right.$ compared to $\left.0.1-0.2 \mathrm{~J} / \mathrm{cm}^{2}\right)$, as well as a higher heat of fusion,,- we expect fluences greater than those required to melt the amorphous skin (i.e., greater than about $1.0 \mathrm{~J} / \mathrm{cm}^{2}$ ) not to produce melting depths extending far beyond the amorphous skin depth, although high fluences could be effective at crystallizing amorphous silicon pockets within the conical surface structures through heat diffusion. The result that additional ns laser pulses do not lead to additional increases in crystallinity suggests that the first ns pulse produces a melting depth that is similar to or greater than the melting depth produced by subsequent ns pulses, which is consistent with the higher melting threshold and latent heat of fusion of crystalline silicon relative to amorphous silicon. The melting depths and dynamics may not depend significantly on the laser wavelength within the range we investigated (193-355 nm), because the optical absorption depth in silicon is fairly uniform in this range $(5-10 \mathrm{~nm}), \frac{53}{2}$ and the amount of crystallization we measured after ns laser annealing did not depend on the ns laser wavelength.

Nanosecond laser annealing also smoothed the surface on the $\mathrm{nm}$ scale while leaving the $10-\mu \mathrm{m}$-scale structures intact. We speculate that nm-scale smoothing could improve conformality during subsequent deposition of electrodes or additional semiconductor layers. Maintaining the $\mu \mathrm{m}$-scale structures, on the other hand, leaves the geometric light-trapping properties of the surface intact (Figure 4). The 1- $\mu \mathrm{m}$-scale conical surface structures made with a fs-laser fluence of $2.5 \mathrm{~kJ} / \mathrm{m}^{2}$, in contrast, were destroyed by high-fluence ns laser annealing $\left(2.2 \mathrm{~J} / \mathrm{cm}^{2}\right)$, which reduced their light-trapping effectiveness (see supplementary material). .44 However, further investigation of ns laser annealing on 1- $\mu \mathrm{m}$-scale conical surface structures could be interesting, because such structures produce considerable light-trapping effects, can be crystallized without being destroyed by using low-fluence ns laser annealing $\left(e . g ., 0.6 \mathrm{~J} / \mathrm{cm}^{2}\right)$, could potentially be fabricated on thin silicon substrates, and could be easier to integrate into devices (e.g., easier to contact electrically) than $10-\mu \mathrm{m}$-scale structures.

Nanosecond laser annealing also reduced the width of the Raman crystalline silicon peak at $520 \mathrm{~cm}^{-1}$ (Figure 3 inset). Lattice stress and small grain sizes can both increase the width of the $520 \mathrm{~cm}^{-1}$ peak as well as shift the peak to lower

wavenumbers. .545 Thus, the reduction in peak width after ns laser annealing could indicate a reduction in lattice stress and/or an increase in grain size. Because the spectral resolution of our Raman measurements is only $2.0 \mathrm{~cm}^{-1}$, peak shifts to lower wavenumbers might result in an apparent increase in peak width. After ns laser annealing, the peak width is still greater than that from a pristine silicon wafer, indicating the presence of residual lattice stress, perhaps from the above-equilibrium concentration of dopant atoms.

The increase in the turn-on voltage of the hyperdoped black silicon diodes after rapid thermal annealing (Figure 5) could be due to the removal of Schottky barriers that form at the metal-semiconductor interface during electrode deposition. 


\section{PREPRINT}

Consistent with this interpretation, the turn-on voltage of $0.2 \pm 0.1 \mathrm{~V}$ we observed after electrode deposition is typical of

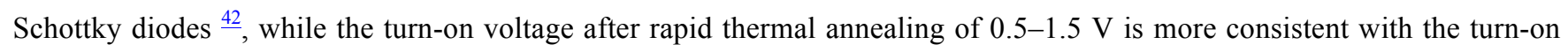
voltage of around $0.7 \mathrm{~V}$ that is typical of silicon homojunction diodes. $\underline{43}$ Furthermore, when rapid thermal annealing was performed before the electrodes were deposited, the turn-on voltage remained at $0.2 \pm 0.1 \mathrm{~V}$, and no changes in crystallinity were detected after rapid thermal annealing, indicating that the increase in turn-on voltage was not due to structural changes in the hyperdoped black silicon itself, but rather changes within the electrode or at the electrode-silicon interface. Indeed, rapid thermal annealing has been reported to reduce significantly the density of defect states at silicon interfaces. $\underline{56}$ The fact that the current-bias curves showed rectification both before and after ns laser annealing (Figure 6) suggests that in hyperdoped black silicon containing amorphous regions, a rectifying junction can still be formed, perhaps in localized crystalline regions.

Nanosecond laser annealing reactivated the sub-bandgap optical absorptance after it was deactivated by thermal annealing, as shown in Figure 7. The deactivation of sub-bandgap optical absorptance, which is associated with an increase in electron mobility and changes in sheet carrier concentration, has been explained using the Johnson-Mehl-AvramiKolmogorov framework describing diffusion-mediated changes in metastable supersaturated solid solutions such as hyperdoped silicon. ${ }^{31}$ However, the reactivation of the sub-bandgap optical absorptance after heating and fast cooling suggests that the degree of departure from room-temperature equilibrium of the dopant atoms, as reflected by the placement of the dopant atoms within the lattice, is reversible and important in describing the properties of hyperdoped silicon, similarly to the way in which fictive temperature is important in describing the properties of glasses — another non-equilibrium material. Reactivation has been previously reported when deactivated hyperdoped silicon was irradiated with fs laser pulses or heated to temperatures above $1350 \mathrm{~K}$ followed by fast cooling (e.g., in silicone oil, yielding estimated cooling rates of about $250 \mathrm{~K} / \mathrm{s}) . \frac{32}{=}$ The reactivation we observe after ns laser annealing, however, is more complete than what has been previously reported and produces or maintains high crystallinity, unlike reactivation by fs laser pulses, which typically produces a layer of amorphous silicon..$^{29}$

We combined thermal and ns laser annealing in sequence to demonstrate the design flexibility provided by combining equilibrium with non-equilibrium processing techniques (Figure 7). In practice, an initial thermal annealing step can be useful for increasing crystallinity or obtaining electrical rectification, $\underline{13}$ but it leads to deactivation of sub-bandgap optical absorptance. $\stackrel{32}{\text { Subsequent }}$ ns laser annealing can then reactivate the optical absorptance while maintaining high crystallinity. Similarly, rapid thermal annealing can be necessary to obtain ohmic contact after electrode deposition, but, again, some deactivation of the optical absorptance occurs. Another application of ns laser annealing can then reactivate the optical 


\section{PREPRINT}

absorptance near to its original level. Overall, we noticed a small drop in above-bandgap absorptance after ns laser annealing of about $5 \%$ absolute due to an increase in surface reflectance, which is probably due to smoothing of the surface, and the final ns laser annealing step removed our metal electrodes. In practice, removal of the electrodes might be avoided by using thicker electrodes, more robust electrode materials, transparent electrodes, or by shielding the electrodes with a mask during ns laser annealing. The combination of thermal and ns laser annealing can produce hyperdoped black silicon that is highly crystalline, highly absorptive to above- and below-bandgap wavelengths, and electrically rectifying. The process we carried out is not a prescription for a specific device, but is meant to be illustrative of the design flexibility provided by combining thermal and ns laser annealing.

We conclude our discussion by commenting on some of the remaining challenges in fabricating hyperdoped black silicon for sub-bandgap optoelectronic devices. The main challenge we address in this work is to obtain high crystallinity and high sub-bandgap optical absorptance concurrently, which we accomplish with ns laser annealing. However, before researchers can expect hyperdoped materials to find widespread use for sub-bandgap optoelectronic devices, some additional challenges need to be addressed. One of those challenges is to obtain doping concentrations within the range needed to produce an intermediate band. In silicon hyperdoped with sulfur, selenium or tellurium, this range is below 0.4 at. \%, with the best figures of merit below 0.1 at. $\% . \underline{57} \underline{23}$ Silicon that is hyperdoped with sulfur, selenium, or tellurium above 0.4 at. $\%$ is metallic and therefore not expected to produce significant optoelectronic (photoconductive or photovoltaic) response. Yet hyperdoped silicon fabricated with ion implantation or with femtosecond laser irradiation often contains dopant concentrations of 1 at. $\%$ or more, including much of the hyperdoped silicon reported in the literature and, likely, the hyperdoped black silicon we examined in this work. It is possible to fabricate hyperdoped silicon with dopant concentrations of $<0.4$ at. \% with fs laser irradiation (by using a low number of pulses and a low pulse fluence $\underline{21} \underline{13}$ ) or with ion implantation (by reducing the implanted ion dose $\stackrel{22}{ }$ ), but the resultant hyperdoped silicon is typically flat, so it does not show light trapping effects and therefore is not a strong optical absorber of sub-bandgap wavelengths. Addressing this challenge will require methods to control the dopant concentration and light-trapping properties of hyperdoped silicon independently.

Another challenge in fabricating hyperdoped black silicon for sub-bandgap optoelectronic devices is in controlling the hyperdoped layer thickness so that charge carriers can be extracted efficiently. Charge carriers in hyperdoped silicon have

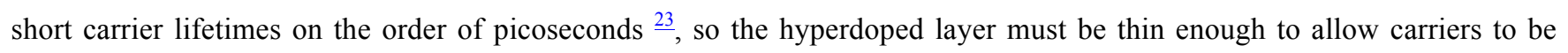
transported out of the layer before recombination occurs. The transport length for holes in sulfur-hyperdoped silicon was estimated in one study to be around $100 \mathrm{~nm}, \frac{25}{5}$ but in general the transport length can be expected to depend on a number of factors, including the dopant concentration, the material microstructure, and electrostatic fields set up by the dopant 


\section{PREPRINT}

concentration gradient. If the electrostatic fields set up by the dopant concentration gradient prove to be a limiting factor in carrier transport, then it might necessary to control the dopant concentration gradient $\underline{58} \underline{59}$ (e.g., producing a monotonic dopant concentration profile with depth) in order to fabricate efficient hyperdoped intermediate band photovoltaics or subbandgap photodetectors. Developing methods for meeting these device requirements are important areas of future research.

\section{CONCLUSION}

Nanosecond laser annealing can crystallize and remove pressure-induced phases from the surface of hyperdoped black silicon while maintaining a light-trapping surface morphology and high sub-bandgap optical absorptance. Additionally, ns laser annealing can reactivate the sub-bandgap optical absorptance after it has been deactivated by thermal annealing. Furthermore, we find that ns laser annealing does not interfere with the formation of rectifying homojunctions in hyperdoped black silicon. Thermal annealing and ns laser annealing can be combined in sequence to produce hyperdoped black silicon that is highly crystalline, shows high above-bandgap and sub-bandgap optical absorptance, and is electrically rectifying. The effects of ns laser irradiation on hyperdoped black silicon are not highly sensitive to laser wavelength, pulse duration, or

number of pulses used. Because ns laser annealing is based on physical processes of melting/heating and fast resolidification/cooling, it could potentially be applied to other non-equilibrium material systems beyond hyperdoped black silicon. The combination of equilibrium processing techniques (e.g., thermal annealing) with non-equilibrium processing techniques (e.g., ns laser annealing) could be a useful approach for controlling the properties of non-equilibrium materials in general.

Fabricating optoelectronic devices using hyperdoped black silicon presents a number of challenges, including concurrently controlling the crystallinity, optical absorptance, dopant concentration, and hyperdoped layer thickness. Here, we use ns laser annealing on hyperdoped black silicon to obtain high crystallinity and optical absorptance while also maintaining electrical rectification. Going forward, developing methods to control the dopant concentration and hyperdoped layer thickness in hyperdoped black silicon will be important for fabricating sub-bandgap optoelectronic devices. 


\section{PREPRINT}

\section{ACKNOWLEDGMENTS}

Several people contributed to the work described in this paper. B.F. conceived of the basic idea for this work. B.F., D.P., H.G., and P.H.R. designed and carried out the experiments and analyzed the results. E.M., M.J.A., and S.G. supervised the research and the development of the manuscript. B.F. wrote the first draft of the manuscript; all authors subsequently took part in the revision process and approved the final copy of the manuscript. Sarah Griesse-Nascimento provided feedback on the manuscript throughout its development. B.F. wishes to thank Thierry Sarnet, Gaëlle Coustillier, David Grojo, and Philippe Delaporte from Le Laboratoire LP3, Marseille, France, for allowing use of their equipment. The research described in this paper was supported by a U.S. National Science Foundation Fellowship and a National Defense Science and Engineering Graduate Fellowship to B.F. and a grant from the Programa Nacional de movilidad de recursos humanos del Plan Nacional I+D+i 2008-2011 of the MEC to D.P. (EX-2010-0662). This work was also supported by the US Air Force Office of Scientific Research (FA9550-14-1-0150) and the US National Science Foundation (DMR-0934480). This work was performed in part at the Center for Nanoscale Systems (CNS), a member of the National Nanotechnology Infrastructure Network (NNIN), which is supported by the National Science Foundation under NSF award no. ECS-0335765. CNS is Part of Harvard University. This work made use of the MRSEC Shared Experimental Facilities at MIT, supported by the U.S. National Science Foundation under award number DMR-1419807.

\section{REFERENCES}

S. H. Pan, D. Recht, S. Charnvanichborikarn, J. S. Williams, and M. J. Aziz, Appl. Phys. Lett. 98, 121913 (2011).

H. M. Branz, V. E. Yost, S. Ward, K. M. Jones, B. To, and P. Stradins, Appl. Phys. Lett. 94, 231121 (2009).

J. E. Carey, C. H. Crouch, M. Shen, and E. Mazur, Optics Letters 30, 1773 (2005).

A. J. Said, D. Recht, J. T. Sullivan, J. M. Warrender, T. Buonassisi, P. D. Persans, and M. J. Aziz, Appl. Phys. Lett. 99, 073503 (2011).

A. Luque, A. Marti, and C. Stanley, Nat. Photonics 6, 146 (2012).

M.-J. Sher, M. T. Winkler, and E. Mazur, MRS Bull. 36, 439 (2011).

C. H. Crouch, J. E. Carey, J. M. Warrender, M. J. Aziz, E. Mazur, and F. Y. Genin, Appl. Phys. Lett. 84, 1850 (2004).

M. Tabbal, T. Kim, D. Woolf, B. Shin, and M. Aziz, Appl. Phys. A 98, 589 (2010).

M. J. Smith, M. T. Winkler, M.-J. Sher, Y.-T. Lin, E. Mazur, and S. Gradečak, Appl. Phys. A 105, 795 (2011).

M. J. Smith, M.-J. Sher, B. Franta, Y.-T. Lin, E. Mazur, and S. Gradecak, Appl. Phys. A 114, 1009 (2014).

T. G. Kim, J. M. Warrender, and M. J. Aziz, Appl. Phys. Lett. 88, 241902 (2006). 


\section{PREPRINT}

B. P. Bob, A. Kohno, S. Charnvanichborikarn, J. M. Warrender, I. Umezu, M. Tabbal, J. S. Williams, and M. J. Aziz, J. Appl. Phys. 107, 123506 (2010).

M. T. Winkler, M.-J. Sher, Y.-T. Lin, M. J. Smith, H. Zhang, S. Gradečak, and E. Mazur, J. Appl. Phys. 111, 093511 (2012).

S. Koynov, M. S. Brandt, and M. Stutzmann, Appl. Phys. Lett. 88, 203107 (2006).

L. L. Ma, Y. C. Zhou, N. Jiang, X. Lu, J. Shao, W. Lu, J. Ge, X. M. Ding, and X. Y. Hou, Appl. Phys. Lett. 88, 171907 (2006).

D. Zielke, D. Sylla, T. Neubert, R. Brendel, and J. Schmidt, IEEE J. Photovolt. 3, 656 (2013).

M. Abbott and J. Cotter, Prog. Photovoltaics 14, 225 (2006).

B. K. Nayak, V. V. Iyengar, and M. C. Gupta, Prog. Photovoltaics 19, 631 (2011).

M. Halbwax, T. Sarnet, P. Delaporte, M. Sentis, H. Etienne, F. Torregrosa, V. Vervisch, I.

Perichaud, and S. Martinuzzi, Thin Solid Films 516, 6791 (2008).

E. Ertekin, M. T. Winkler, D. Recht, A. J. Said, M. J. Aziz, T. Buonassisi, and J. C. Grossman, Phys. Rev. Lett. 108, 026401 (2012).

M. T. Winkler, D. Recht, M.-J. Sher, A. J. Said, E. Mazur, and M. J. Aziz, Phys. Rev. Lett. 106, 178701 (2011).

J. T. Sullivan, R. G. Wilks, M. T. Winkler, L. Weinhardt, D. Recht, A. J. Said, B. K. Newman, Y. Zhang, M. Blum, S. Krause, W. L. Yang, C. Heske, M. J. Aziz, M. Bär, and T. Buonassisi, Appl. Phys. Lett. 99, 142102 (2011).

M.-J. Sher, C. B. Simmons, J. J. Krich, A. J. Akey, M. T. Winkler, D. Recht, T. Buonassisi, M. J. Aziz, and A. M. Lindenberg, Appl. Phys. Lett 105, 053905 (2014).

C. B. Simmons, A. J. Akey, J. P. Mailoa, D. Recht, M. J. Aziz, and T. Buonassisi, Adv. Funct. Mater. 24, 2852 (2014).

P. D. Persans, N. E. Berry, D. Recht, D. Hutchinson, H. Peterson, J. Clark, S.

Charnvanichborikarn, J. S. Williams, A. DiFranzo, M. J. Aziz, and J. M. Warrender, Appl. Phys. Lett. 101, 111105 (2012).

I. Umezu, J. M. Warrender, S. Charnvanichborikarn, A. Kohno, J. S. Williams, M. Tabbal, D. G. Papazoglou, X.-C. Zhang, and M. J. Aziz, J. Appl. Phys. 113, 213501 (2013).

M. A. Sheehy, L. Winston, J. E. Carey, C. M. Friend, and E. Mazur, Chem. Mater. 17, 3582 (2005).

M. J. Smith, Y.-T. Lin, M.-J. Sher, M. T. Winkler, E. Mazur, and S. Gradečak, J. Appl. Phys. 110, 053524 (2011).

M. J. Smith, M.-J. Sher, B. Franta, Y.-T. Lin, E. Mazur, and S. Gradečak, J. Appl. Phys. 112, 083518 (2012).

C. H. Crouch, J. E. Carey, M. Shen, E. Mazur, and F. Y. Génin, Appl. Phys. A 79, 1635 (2004).

C. B. Simmons, A. J. Akey, J. J. Krich, J. T. Sullivan, D. Recht, M. J. Aziz, and T. Buonassisi, J. Appl. Phys. 114, 243514 (2013).

B. K. Newman, M.-J. Sher, E. Mazur, and T. Buonassisi, Appl. Phys. Lett. 98, 251905 (2011). J. P. Mailoa, A. J. Akey, C. B. Simmons, D. Hutchinson, J. Mathews, J. T. Sullivan, D. Recht, M. T. Winkler, J. S. Williams, J. M. Warrender, P. D. Persans, M. J. Aziz, and T. Buonassisi, Nat. Commun. 5, 3011 (2014).

K.-M. Guenther, T. Gimpel, J. W. Tomm, S. Winter, A. Ruibys, S. Kontermann, and W. Schade, Applied Physics Letters 104, 042107 (2014).

M. J. Aziz, Metall. Mater. Trans. A 27, 671 (1996).

J. A. Kittl, P. G. Sanders, M. J. Aziz, D. P. Brunco, and M. O. Thompson, Acta Mater. 48, 4797 (2000).

R. L. C. Vink, G. T. Barkema, and W. F. van der Weg, Phys. Rev. B 63, 115210 (2001).

H. Richter, Z. P. Wang, and L. Ley, Solid State Commun. 39, 625 (1981). 
J. Mayer, L. A. Giannuzzi, T. Kamino, and J. Michael, MRS Bull. 32, 400 (2007).

B. R. Tull, J. E. Carey, M. A. Sheehy, C. Friend, and E. Mazur, Appl. Phys. A 83, 341 (2006).

N. G. Semaltianos, S. Logothetidis, W. Perrie, S. Romani, R. J. Potter, S. P. Edwardson, P. French, M. Sharp, G. Dearden, and K. G. Watkins, J. Nanopart. Res. 12, 573 (2010).

H. C. Card and E. H. Rhoderick, J. Phys. D: Appl. Phys. 4, 1589 (1971).

G. L. Pearson and B. Sawyer, Proc. IRE 40, 1348 (1952).

See supplementary material at [URL inserted by AIP Publishing] for figures showing effects of ns laser annealing on hyperdoped black silicon with 1- $\mu \mathrm{m}$-scale surface structures.

M. O. Thompson, J. W. Mayer, A. G. Cullis, H. C. Webber, N. G. Chew, J. M. Poate, and D. C. Jacobson, Phys. Rev. Lett. 50, 896 (1983).

A. G. Cullis, D. T. J. Hurle, H. C. Webber, N. G. Chew, J. M. Poate, P. Baeri, and G. Foti, Appl. Phys. Lett. 38, 642 (1981).

A. J. Akey, D. Recht, J. S. Williams, M. J. Aziz, and T. Buonassisi, Adv. Funct. Mater., doi: 10.1002/adfm.201501450 (2015).

M. O. Thompson, G. J. Galvin, J. W. Mayer, P. S. Peercy, J. M. Poate, D. C. Jacobson, A. G. Cullis, and N. G. Chew, Phys. Rev. Lett. 52, 2360 (1984).

G. Haberfehlner, M. J. Smith, J.-C. Idrobo, G. Auvert, M.-J. Sher, M. T. Winkler, E. Mazur, N. Gambacorti, S. Gradečak, and P. Bleuet, Microscopy and Microanalysis 19, 716 (2013).

J. Förster and H. Vogt, in Excimer-Laser Annealing of Amorphous Silicon Layers, Proceedings of the 2011 COMSOL Conference, Stuttgart, Germany, 2011.

J. Narayan, C. W. White, M. J. Aziz, B. Stritzker, and A. Walthuis, J. Appl. Phys. 57, 564 (1985).

C. J. Shih, C. H. Fang, C. C. Lu, M. H. Wang, M. H. Lee, and C. W. Lan, J. Appl. Phys. 100, 053504 (2006).

M. A. Green and M. J. Keevers, Prog. Photovoltaics 3, 189 (1995).

N. H. Nickel, Laser Crystallization of Silicon-Fundamentals to Devices, Vol. 75 (Academic Press, 2003).

I. De Wolf, Semiconductor Science and Technology 11, 139 (1996).

Y. Ma, T. Yasuda, and G. Lucovsky, J. Vac. Sci. Technol. A 11, 952 (1993).

J. T. Sullivan, C. B. Simmons, J. J. Krich, A. J. Akey, D. Recht, M. J. Aziz, and T. Buonassisi, J. Appl. Phys. 114, 103701 (2013).

Y.-T. Lin, N. Mangan, S. Marbach, T. M. Schneider, G. Deng, S. Zhou, M. P. Brenner, and E. Mazur, Appl. Phys. Lett. 106, 062105 (2015).

M.-J. Sher, N. M. Mangan, M. J. Smith, Y.-T. Lin, S. Marbach, T. M. Schneider, S. Gradečak, M. P. Brenner, and E. Mazur, J. Appl. Phys. 117, 125301 (2015). 


\section{PREPRINT}
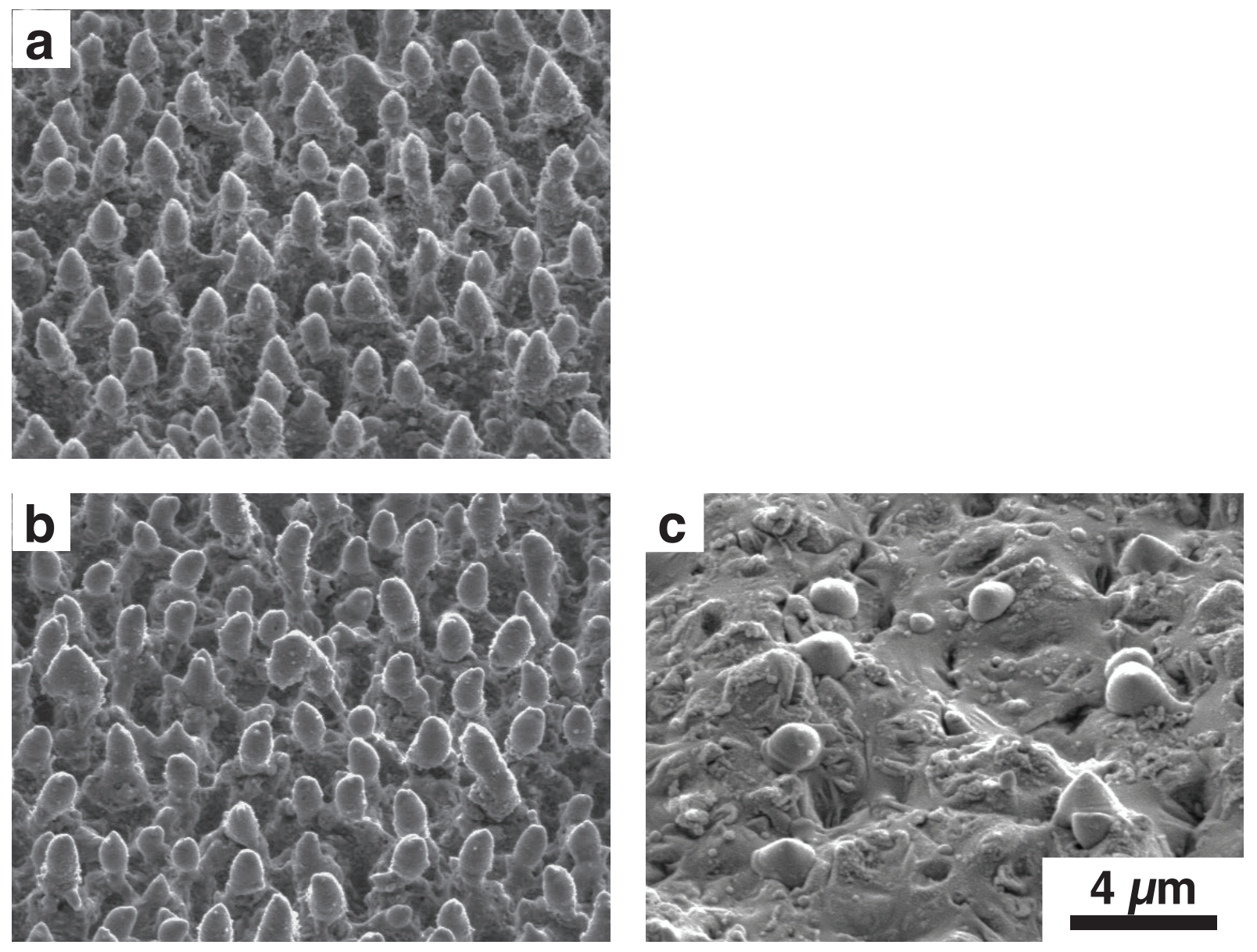

SUPPLEMENTARY FIG. S1. (A) Hyperdoped black silicon (fabricated with a fs-laser pulse fluence of

$2.5 \mathrm{~kJ} / \mathrm{m}^{2}$ ) with light-trapping conical surface structures about $1 \mu \mathrm{m}$ in size. (Image obtained at 45 degree angle without foreshortening correction). (B) Nanosecond laser annealing at low fluences (here, $0.6 \mathrm{~J} / \mathrm{cm}^{2}$ ) kept the surface structures intact while crystallizing the surface. (C) Nanosecond laser annealing at high fluences (here, $2.2 \mathrm{~J} / \mathrm{cm}^{2}$ ) destroyed the surface structures. 
PREPRINT

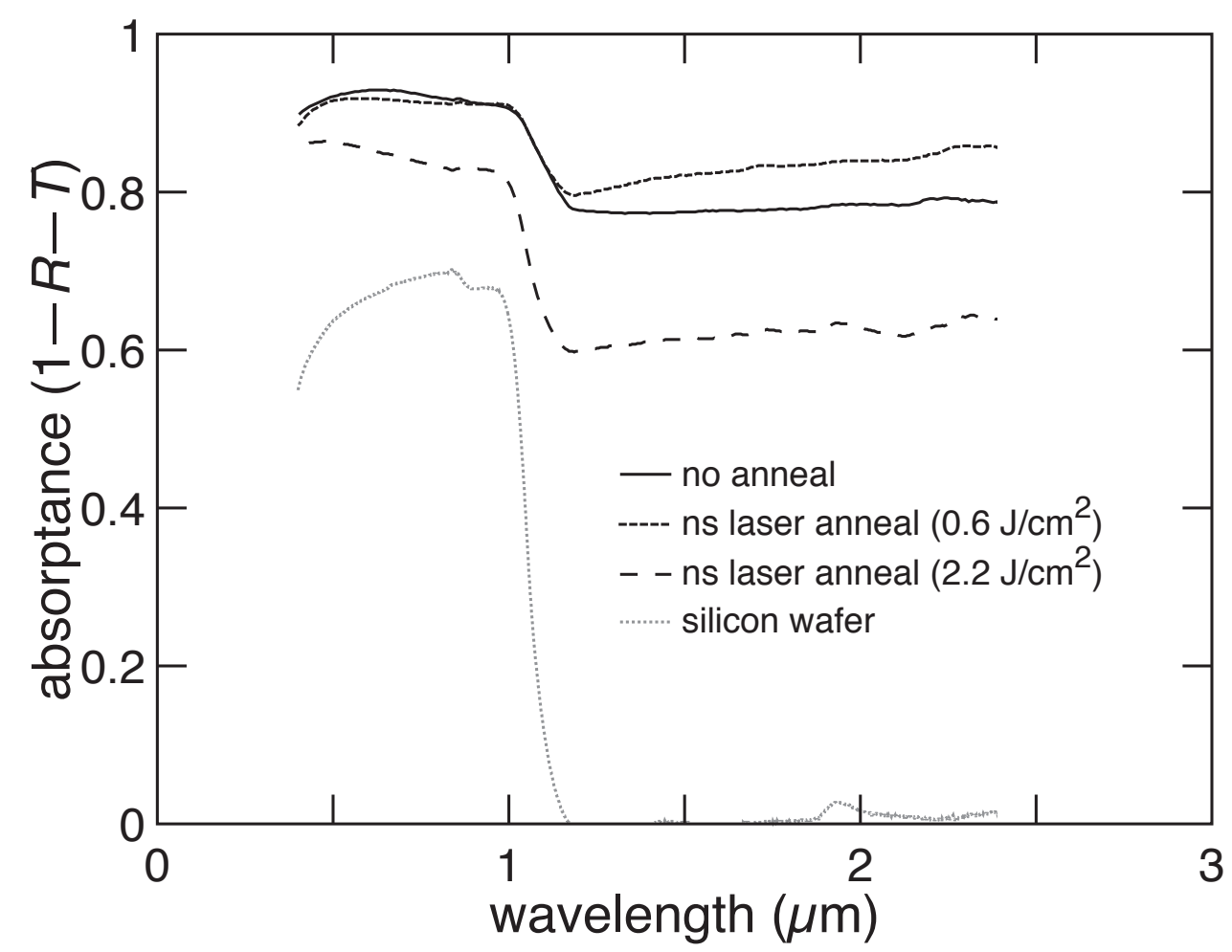

SUPPLEMENTARY FIG. S2. Nanosecond laser annealing at low fluences $\left(e . g ., 0.6 \mathrm{~J} / \mathrm{cm}^{2}\right)$ on $1-\mu \mathrm{m}$ scale surface structures maintained or increased the optical absorptance, but annealing at high fluences (e.g., $2.2 \mathrm{~J} / \mathrm{cm}^{2}$ ) decreased the optical absorptance due to the removal of the light-trapping surface structures. 